\title{
FOXA2 is a sensitive and specific marker for small cell neuroendocrine carcinoma of the prostate
}

\author{
Jung Wook Park ${ }^{1}$, John K Lee ${ }^{2,3}$, Owen N Witte ${ }^{1,4,5}$ and Jiaoti Huang6 \\ ${ }^{1}$ Department of Microbiology, Immunology and Molecular Genetics, David Geffen School of Medicine, \\ University of California-Los Angeles, Los Angeles, CA, USA; ${ }^{2}$ Division of Hematology and Oncology, \\ Department of Medicine, David Geffen School of Medicine, University of California-Los Angeles, Los \\ Angeles, CA, USA; ${ }^{3}$ Molecular Biology Institute, David Geffen School of Medicine, University of California- \\ Los Angeles, Los Angeles, CA, USA; ${ }^{4}$ Eli and Edythe Broad Center of Regenerative Medicine and Stem Cell \\ Research, University of California-Los Angeles, Los Angeles, CA, USA; ${ }^{5}$ Department of Molecular and \\ Medical Pharmacology, David Geffen School of Medicine, University of California-Los Angeles, Los Angeles, \\ CA, USA and ${ }^{6}$ Department of Pathology, Duke University School of Medicine, Durham, NC, USA
}

\begin{abstract}
The median survival of patients with small cell neuroendocrine carcinoma is significantly shorter than that of patients with classic acinar-type adenocarcinoma. Small cell neuroendocrine carcinoma is traditionally diagnosed based on histologic features because expression of current immunohistochemical markers is inconsistent. This is a challenging diagnosis even for expert pathologists and particularly so for pathologists who do not specialize in prostate cancer. New biomarkers to aid in the diagnosis of small cell neuroendocrine carcinoma are therefore urgently needed. We discovered that FOXA2, a pioneer transcription factor, is frequently and specifically expressed in small cell neuroendocrine carcinoma compared with prostate adenocarcinoma from published mRNA-sequencing data of a wide range of human prostate cancers. We verified the expression of FOXA2 in human prostate cancer cell lines and xenografts, patient biopsy specimens, tissue microarrays of prostate cancers with lymph node metastasis, primary small cell neuroendocrine carcinoma, and metastatic treatment-related small cell neuroendocrine carcinoma and cases from a rapid autopsy program. FOXA2 expression was present in $\mathrm{NCl}-\mathrm{H} 660$ and PC3 neuroendocrine cell lines, but not in LNCAP and CWR22 adenocarcinoma cell lines. Of the human prostate cancer specimens, 20 of 235 specimens (8.5\%) showed diagnostic histologic features of small cell neuroendocrine carcinoma as judged histologically. Fifteen of 20 small cell neuroendocrine carcinoma tissues $(75 \%)$ showed strong expression of FOXA2 (staining intensity 2 or 3). FOXA2 expression was also detected in 9 of 215 prostate cancer tissues (4.2\%) that were histologically defined as adenocarcinoma. Our findings demonstrate that FOXA2 is a sensitive and specific molecular marker that may be extremely valuable in the pathologic diagnosis of small cell neuroendocrine carcinoma. Modern Pathology (2017) 30, 1262-1272; doi:10.1038/modpathol.2017.44; published online 16 June 2017
\end{abstract}

Small cell neuroendocrine carcinoma of the prostate is thought to represent $2 \%$ of all primary prostate cancers. ${ }^{1}$ Recently, it is now widely recognized that a significant proportion of patients develop small cell neuroendocrine carcinoma after failing hormonal therapy for prostatic adenocarcinoma. ${ }^{2,3}$ Autopsy

Correspondence: Professor J Huang, MD, PhD, Department of Pathology, Duke University School of Medicine, Durham, NC 27710, USA or Professor ON Witte, MD, Department of Microbiology, Immunology and Molecular Genetics, David Geffen School of Medicine, University of California-Los Angeles, Los Angeles, CA 90024, USA.

E-mail: Jiaoti.huang@duke.edu or Owenwitte@mednet.ucla.edu Received 2 October 2016; revised 5 April 2017; accepted 6 April 2017; published online 16 June 2017 series indicate that $10-20 \%$ of patients with lethal castration-resistant prostate cancer should be classified as small cell neuroendocrine carcinoma. ${ }^{4}$ Small cell neuroendocrine carcinoma may be pure but in up to $50 \%$ of cases it is associated with conventional prostatic adenocarcinoma. ${ }^{2,3}$ Because small cell neuroendocrine carcinoma is independent of androgen receptor signaling and insensitive to hormonal therapy, chemotherapy and radiation therapy are the only available palliative treatment modalities for this disease. ${ }^{5}$ No curative therapies exist for patients with small cell neuroendocrine carcinoma due to a lack of understanding of the molecular basis of the disease.

Small cell neuroendocrine carcinoma has aggressive clinical behavior such as early metastasis. Some 
patients show increased serum levels of chromogranin A, neuron-specific enolase, bombesin, 5-hydroxytryptamine, and gastrin ${ }^{6}$ but the serum markers are unreliable for a definitive diagnosis. Small cell neuroendocrine carcinoma is diagnosed histologically based on characteristic morphologic features such as scant cytoplasm, darkly stained nuclei with finely granular 'salt and pepper' chromatin pattern, absent nucleoli, nuclear molding, tumor necrosis, and frequent mitotic figures. Unfortunately, there is significant overlap between small cell neuroendocrine carcinoma and poorly differentiated adenocarcinoma (ie, Gleason 5 pattern) making histologic diagnosis challenging. As a general practice, pathologists often use ancillary studies particularly immunohistochemical staining to help them confirm the diagnosis of difficult cases. Small cell neuroendocrine carcinoma often shows expression of neuroendocrine differentiation markers chromogranin A and synaptophysin, loss of androgen receptor (AR) and prostate specific antigen (PSA). However, expression of these markers shows significant variability from case to case and pathologists still consider histology the gold standard. ${ }^{7}$ Given these challenges in diagnosing small cell neuroendocrine carcinoma and distinguishing it from high-grade adenocarcinoma, sensitive, and specific molecular markers are urgently needed.

The genetically engineered TRAMP mouse model is a classic model of small cell neuroendocrine carcinoma. ${ }^{8}$ In this model, the lineage-specific transcription factor FOXA2 was exclusively expressed in synaptophysin-positive tumor cells of small cell neuroendocrine carcinoma. We recently reported that enforced expression of N-Myc and activated AKT1 drives benign human prostate epithelial cells to small cell neuroendocrine carcinoma and FOXA2 expression was uniformly identified in these tumors. ${ }^{9}$ In contrast, during normal prostate development in mouse, FOXA2 is expressed early in embryonic prostate epithelial bud formation but disappears later except in a small proportion of basal epithelial cells within the peri-urethral ducts of the mature prostate. ${ }^{10}$ In human prostate tissue, FOXA2 expression is focally detected in a subset of basal cells which also express synaptophysin. ${ }^{11}$ This FOXA2 expression was found in two different morphological types of prostate neuroendocrine cells. ${ }^{12,13}$

In the present study, we assessed expression of FOXA2 in mRNA expression data sets of human prostate cancers and a panel of prostate cancer cell lines as well as by immunohistochemical staining in multiple cohorts of human prostate cancer. FOXA2 expression was not detectable in primary and metastatic prostate adenocarcinomas, whereas it was highly expressed in small cell neuroendocrine carcinoma. In addition, FOXA2 expression was elevated in metastatic castration-resistant prostate cancer samples that were histologically classified as small cell neuroendocrine carcinoma. Our findings confirm that assessment of FOXA2 expression in clinical prostate cancer tissue is a valuable tool in the diagnosis and differential diagnosis of small cell neuroendocrine carcinoma.

\section{Materials and methods}

\section{Tissue Microarrays}

Several tissue microarrays were constructed and have been reported previously. A tissue microarray of treatment-naive samples was constructed with prostatectomy specimens including prostate cancer and the adjacent benign prostate tissue. ${ }^{14,15}$ Three cores were obtained from benign and cancer areas of each of the prostatectomy specimens and constructed into tissue microarrays. A tissue microarray of treatment-naive prostate cancer with lymph node metastasis was constructed from 22 cases of prostatectomy specimens. Castration-resistant prostate cancer tissue microarray was built using castration-resistant prostate cancer tissue from 16 patients. These patients had primary adenocarcinoma of the prostate but were treated with hormonal therapy instead of surgery. Eventually, the tumor recurred (castration-resistant prostate cancer) and caused urinary obstruction. Transurethral resection of the prostate was performed to relieve the obstructive symptoms and tissues were histologically diagnosed as adenocarcinoma and constructed into a tissue microarray of castration-resistant prostate cancer. ${ }^{16,17}$ A small cell neuroendocrine carcinoma tissue microarray was constructed from primary small cell neuroendocrine carcinoma cases.

\section{Immunohistochemistry}

Serial 4- $\mu \mathrm{m}$-thick sections were de-paraffinized, and endogenous peroxidase activity was blocked with $3 \%$ $\mathrm{H}_{2} \mathrm{O}_{2}$ in methanol. Antigen was retrieved by steam heating in $0.01 \mathrm{M}$ citrate buffer ( $\mathrm{pH}$ 6.0) for $30 \mathrm{~min}$ in a commercial vegetable steamer. Nonspecific binding was blocked with $5 \%$ horse serum in low-salt TBST medium (0.02 M Tris, $0.13 \mathrm{M} \mathrm{NaCl,} 0.02 \%$ Tween 20 (pH 7.8)), and whole-tissue sections were incubated overnight at $4{ }^{\circ} \mathrm{C}$ with anti-rabbit (or mouse) HPR conjugated antibody. Subsequently, sections were processed for immunohistochemistry by using ABC system (Dako) according to the manufacturer's instructions. Sections were counterstained with hematoxylin, dehydrated, and mounted.

\section{Antibodies}

Antibodies used for immunohistochemistry and immunoblot analysis included FOXA1 (1:200 dilution, ab170933, Abcam), FOXA2 (1:200 dilution, ab108422, Abcam), Synaptophysin (1:200 dilution, IR66061-2, Dako), Chromogranin A (1:100 dilution, M086929-2, Dako), and GAPDH (1:10 000 dilution, GTX627408, Genetex). 


\section{Quick-Score System}

Quick-score was calculated ${ }^{18}$ as reported, the intensity of staining is multiplied by the percentage of staining to derive a composite score (a range from 0 to 300$)$.

\section{Xenograft}

$1.0 \times 10^{6}$ cells of LNCaP, CWR22, PC3, and NCI-H660 cell lines were subcutaneous injected with Matrigel (Corning) into NSG (NOD.Cg-Prkdc ${ }^{\text {scid }}$ Il2rg $^{\text {tm1Wjl/ }}$ $\mathrm{SzJ}^{19}$ ) mice. Xenografts were harvested once they reached $1 \mathrm{~cm}$ in diameter.

\section{Animal Work}

NOD.Cg-PrkdC ${ }^{\text {scid }}$ Il2rg ${ }^{\text {tm1Wjl} / S z J ~(N S G) ~ m i c e ~ w e r e ~}$ originally purchased from the Jackson Laboratories and were housed and bred under the care of the Division of Laboratory Animal Medicine at the University of California, Los Angeles (UCLA). Subcutaneous injection was performed according to protocols approved by UCLA's Animal Research Committee.

\section{Results}

FOXA2 is Selectively Expressed in Human Small Cell Neuroendocrine Carcinoma

Recent studies have shown that small cell neuroendocrine carcinoma has a distinct transcriptional profile compared with prostate adenocarcinoma. ${ }^{20-23} \mathrm{We}$ analyzed three published high-throughput RNAsequencing data sets containing prostate adenocarcinoma and small cell neuroendocrine carcinoma samples ${ }^{20-22}$ to identify differentially expressed genes in these tumor subtypes. We identified the upregulation of known neuroendocrine marker genes: neural cell adhesion molecule 1, chromogranin A, and synaptophysin (Figure 1a). We also observed that FOXA2 gene (forkhead box A2) was highly and specifically expressed in the small cell neuroendocrine carcinoma samples (Figure 1a). A previous study molecularly characterized a patientderived xenograft that transdifferentiated from hormone-naïve adenocarcinoma (LTL331) to castrationresistant small cell neuroendocrine carcinoma after relapse (LTL331R). ${ }^{23}$ Their gene expression data similarly showed that androgen-related genes such as $A R, K L K 3$, TMPRSS2, and ERG were downregulated, whereas neuroendocrine-related genes, SYP, CHGA, and NCAM1 were upregulated during the trans-differentiation. ${ }^{23}$ Interestingly, expression of FOXA2 mRNA was highly induced in the relapsed small cell neuroendocrine carcinoma xenograft (Figure 1b). Further confirmation of these findings comes from a recent, larger study that also demonstrated upregulation of FOXA2 expression in small cell neuroendocrine carcinoma compared with castration-resistant prostate adenocarcinoma. ${ }^{22}$

FOXA2 is Specifically Expressed in Human Small Cell Neuroendocrine Carcinoma Cell Lines and Xenografts

To validate the gene expression findings from the analysis of published transcriptome data sets, we evaluated protein levels of FOXA2 in a panel of human prostate cancer cell lines by immunoblot analysis. We found that FOXA2 was exclusively expressed in two neuroendocrine prostate cancer cell lines, PC3 ${ }^{24}$ and NCI-H660, ${ }^{25}$ but not in the prostate adenocarcinoma cell lines, VCaP, LAPC4, 22Rv1, Du145, C4-2, and LNCaP (Figure 2a). RWPE1, an immortalized normal prostate epithelial cell line, ${ }^{26}$ did not express FOXA2 either (Figure 2a). The specificity of the FOXA2 antibody was validated using LNCaP cells transfected with a vector expressing FOXA2 cDNA (Supplementary Figure 1). Consistent with these findings, FOXA2 mRNA is upregulated in PC3 and NCI-H660 based on data from the Cancer Cell Line Encyclopedia as presented in cBioportal (http://www.cbioportal.org) (Figure 2b).

To further assess the distribution and localization of FOXA2 expression in prostate cancer, we generated cell line xenografts of PC3, NCI-H660, LNCaP, and CWR22 in immune-deficient NSG (NOD.CgPrkdc ${ }^{\text {scid }}$ Il2rg ${ }^{\text {tm1Wjl }} / \mathrm{SzJ}$ ) mice. ${ }^{19}$ Immunohistochemistry for FOXA2 in PC3 and NCI-H660 xenograft sections displayed strong nuclear expression, while no expression was found in LNCaP and CWR22 xenografts (Figure 2c). The expression pattern of FOXA2 was homogeneous in the xenografts of the two neuroendocrine prostate cancer cell lines. Our analysis of human prostate cancer cell lines confirmed that FOXA2 is specifically expressed in small cell neuroendocrine carcinoma.

\section{Primary Human Small Cell Neuroendocrine Carcinoma Tissues Express High Levels of FOXA2}

We next assessed FOXA2 expression in a large panel of human prostate cancer tissues. We performed immunohistochemistry for FOXA2 on multiple tissue microarrays of benign prostate, primary treatment-naive human prostate cancers including prostate adenocarcinoma without metastasis (primary adenocarcinoma), prostate adenocarcinoma with lymph node metastasis (LN+ adenocarcinoma), and primary small cell neuroendocrine carcinoma. The intensity and percentage of positive FOXA2 staining were scored by the pathologist who was blinded to the diagnosis. The majority of benign prostate tissues showed no detectable FOXA2 staining and only 11 of 149 benign tissues (7.4\%) displayed focal FOXA2 staining (Figure 3a). This focal staining of FOXA2 was previously reported in a subset of basal cells expressing synaptophysin. ${ }^{11}$ Consistent with the previous findings, we also found 
a

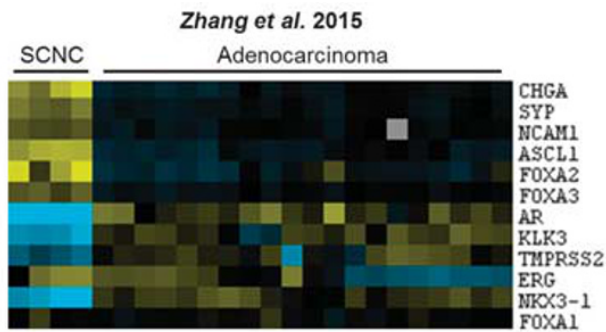

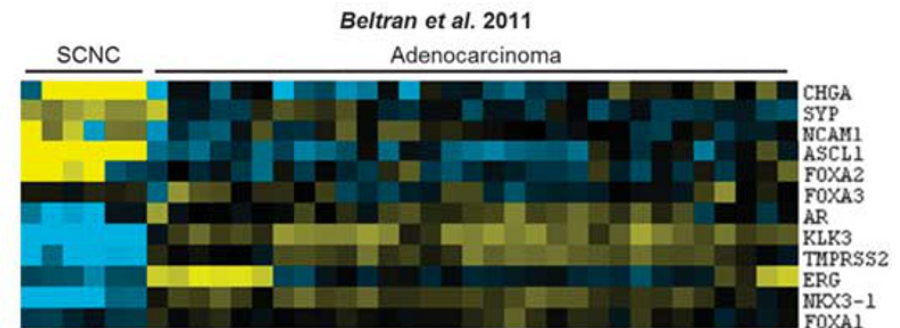

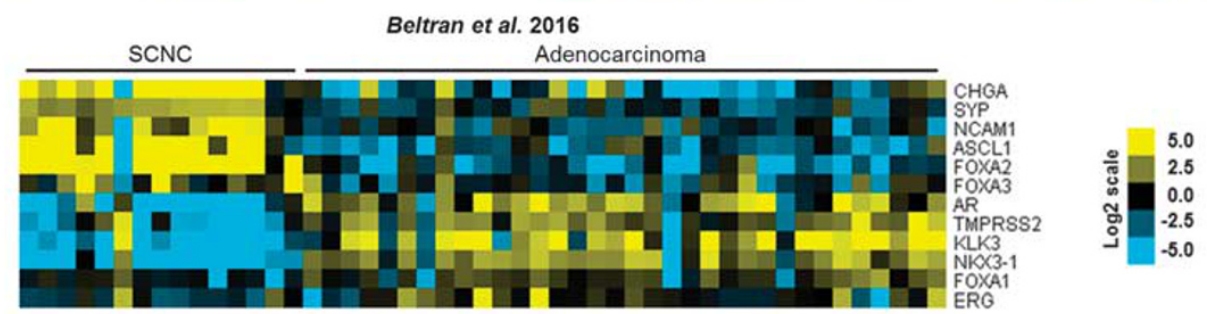

b

Akamatsu et al. 2015
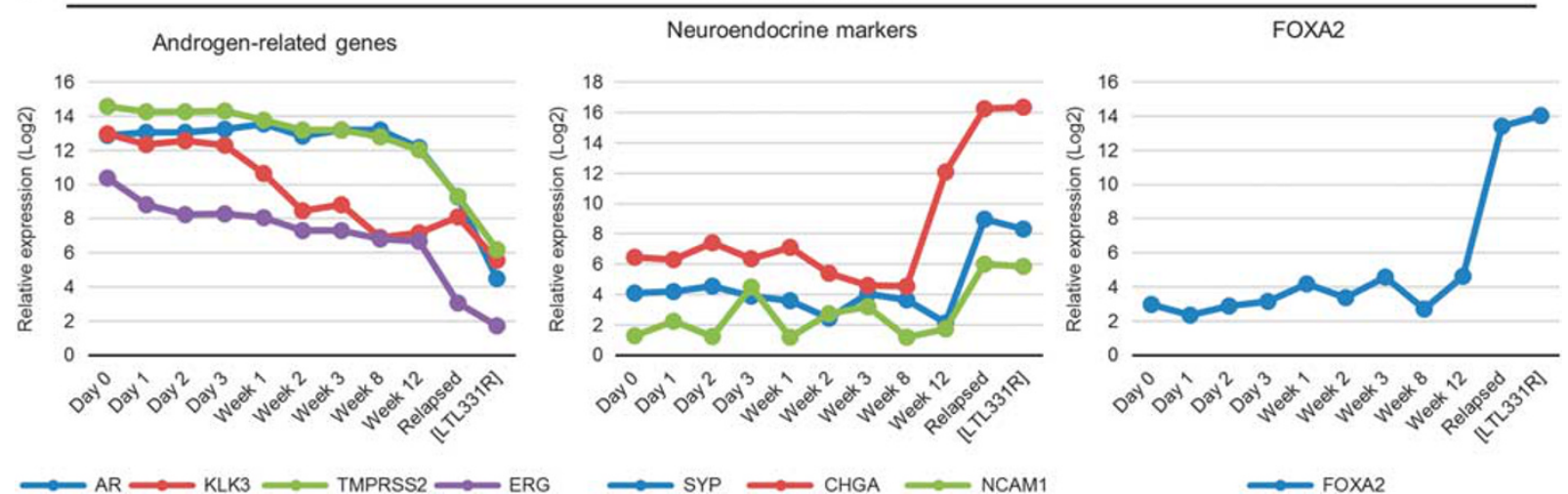

Figure 1 Expression of FOXA2 mRNA is upregulated in human small cell neuroendocrine carcinoma. (a) Heatmap of a selection of genes in data sets of Beltran et al, ${ }^{20}$ Zhang et al, ${ }^{21}$ and Beltran et al ${ }^{22}$ (contrast; log2 scale). (b) Relative expression of androgen-related genes (AR, KLK3, TMPRSS2, and ERG), Neuroendocrine-related genes (SYP, CHGA, and NCAM1), and FOXA2 at the time points after xenografting of LTL331 specimen in castrated mice in Akamatsu et al. ${ }^{23}$ LTL331R is a castrated resistant xenograft. SCNC, small cell neuroendocrine carcinoma.

that FOXA2 positive cells in benign prostate epithelial cells displayed expression of a neuroendocrine marker, synaptophysin (Supplementary Figure 2). In primary prostate adenocarcinomas (Gleason 6-10) without evidence of metastasis at the time of surgery, only 8 of 143 tissues $(5.6 \%)$ demonstrated positive FOXA2 staining with significant variation in staining intensity between these samples (Figure 3a). None of the prostate adenocarcinomas with lymph node metastasis $(N=22)$ showed FOXA2 expression. Strikingly, all of the small cell neuroendocrine carcinoma tissues $(N=11)$ exhibited strong FOXA2 staining. Seven of 11 small cell neuroendocrine carcinoma tissues $(63.6 \%)$ showed a high intensity (intensity 3, on a scale of 1 to 3 ) of FOXA2 staining (Figure 3a), whereas we only found that only one out of 11 high-grade primary prostate adenocarcinoma (Gleason 8 and 9-10) showed focal FOXA2 positivity with intensity 2 (Figure 3b). In addition, we utilized the Quick-score system ${ }^{18}$ in which the intensity of staining is multiplied by the percentage of staining to derive a composite score. The mean FOXA2 Quick-score in small cell neuroendocrine carcinoma was significantly higher than that of all other prostate adenocarcinomas analyzed $(P<0.05$, Holm-Sidak's multiple comparisons test in Figure 3c). Our results confirm that FOXA2 is relatively specifically expressed in primary human small cell neuroendocrine carcinoma.

\section{Treatment-Related Metastatic Small Cell Neuroendocrine Carcinoma Highly Expresses FOXA2}

While small cell neuroendocrine carcinoma is occasionally seen as a primary tumor, it more commonly occurs as a recurrent tumor in patients who have failed hormonal therapy for primary adenocarcinoma of the prostate. The results shown above have demonstrated that FOXA2 is specifically expressed in primary small cell neuroendocrine carcinoma of treatment-naive patients. We then asked whether FOXA2 may also be a marker of treatment-related metastatic small cell neuroendocrine carcinoma. After hormonal therapy for prostate cancer, the tumor may occur as adenocarcinoma 
(castration-resistant prostate cancer) or small cell neuroendocrine carcinoma. We first performed immunohistochemistry using a tissue microarray containing castration-resistant prostate cancer from 16 patients. These patients had primary adenocarcinoma of the prostate but were treated with hormonal therapy instead of surgery. Eventually, the tumor recurred (castration-resistant prostate cancer) and caused urinary obstruction. Transurethral resection of the prostate was performed to relieve the obstructive symptoms and tissues were histologically diagnosed as adenocarcinoma and constructed into a tissue microarray of castration-resistant prostate cancer. Only one of the cases in the castration-resistant prostate cancer tissue microarray showed FOXA2 expression (staining intensity 2, on a scale of 1 to 3 ) while the remaining 15 tissues demonstrated no FOXA2 expression (Figure 4a).

We also evaluated FOXA2 staining on 41 metastatic castration-resistant prostate cancer samples involving distant sites from 18 different patients obtained at rapid autopsy. The hematoxylin and eosin (H\&E) stained slides were first examined histologically and 8 of the 41 metastatic castrationresistant prostate cancer tissues $(19.5 \%)$ were classified as small cell neuroendocrine carcinoma while the rest had classic features of adenocarcinoma. There was no significant difference in terms of the anatomical locations (Figure 4b). Notably, all small cell neuroendocrine carcinomas were found to be FOXA2 positive while adenocarcinoma cases were all negative. Among the small cell neuroendocrine carcinoma
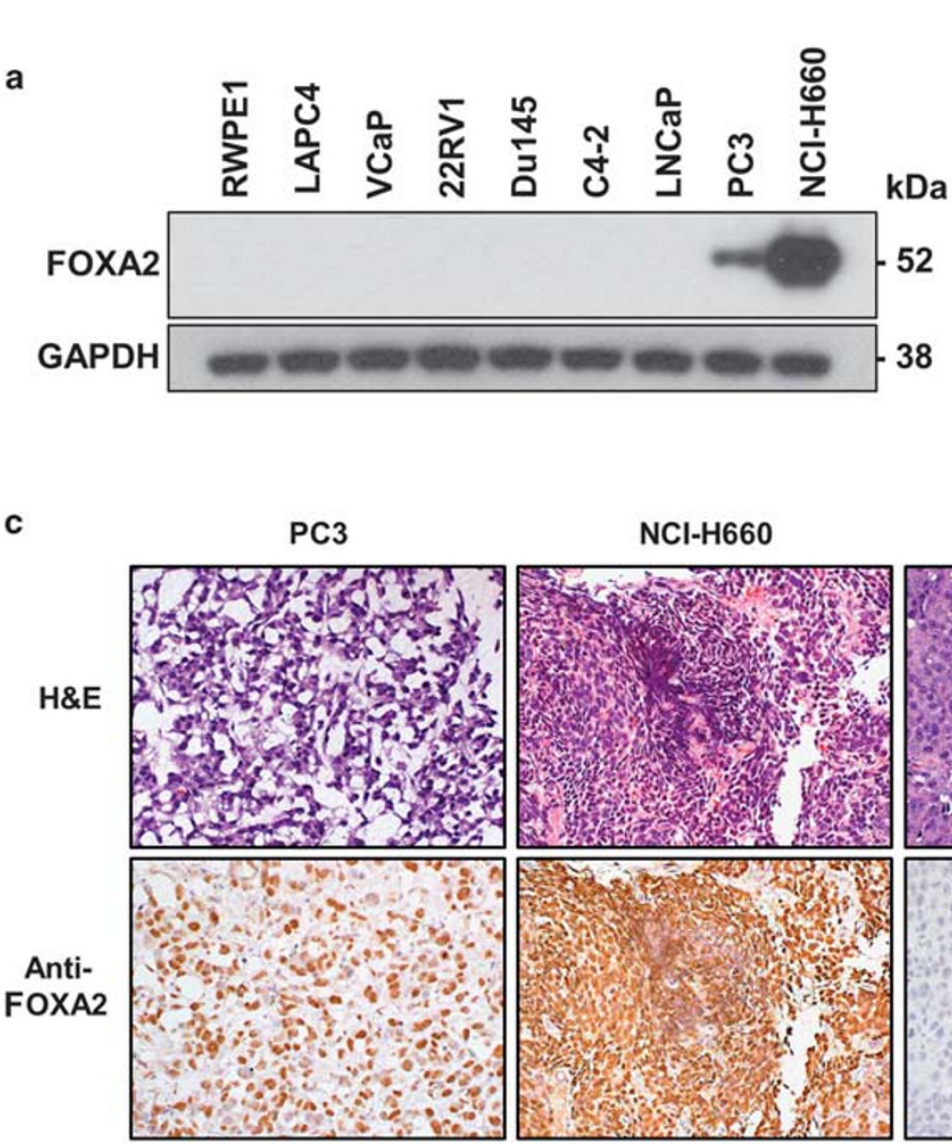

$\mathrm{NCl}-\mathrm{H} 660$
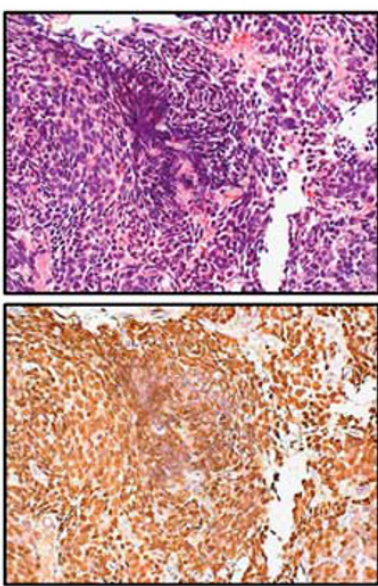

b

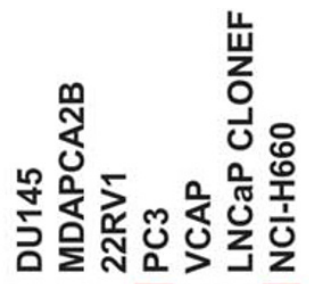

FOXA2

mRNA up-regulation

$z$-score threshold $\pm: 1$
LNCaP

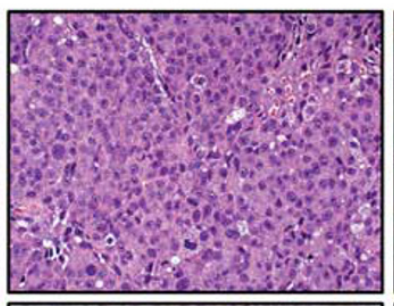

CWR22

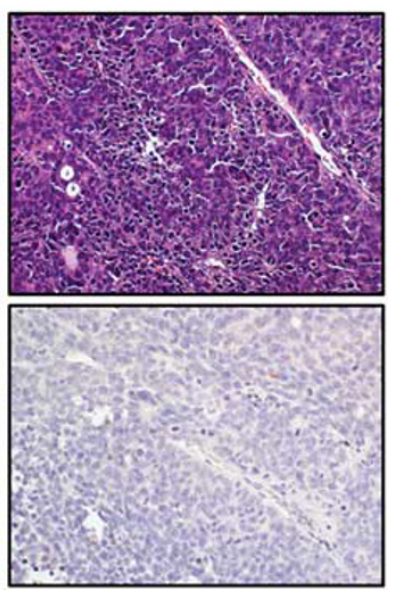

Figure 2 Small cell neuroendocrine carcinoma cell lines specifically express FOXA2. (a) FOXA2 mRNA expression in the Cancer Cell Line Encyclopedia as presented in cBioportal. (b) Immunoblot analysis of FOXA2 in prostate cancer cell lines and an immortalized prostate epithelial cell line (RWPE1). GAPDH is assessed as a loading control. (c) Hematoxylin and eosin staining (H\&E) and immunohistochemical analysis of cell line xenografts of FOXA2 in PC3, NCI-H660, LNCaP, and CWR22. Scale bar, $100 \mu \mathrm{m}$.

Figure 3 FOXA2 expression in treatment-naive prostate cancers. (a) Representative images of FOXA2 immunohistochemistry staining intensity (from 0, no staining to 3, the strongest staining) on the tissue microarrays including benign prostate tissue (Benign Prostate), primary prostate adenocarcinoma (Primary Adenocarcinoma) with Gleason score 3+3, 3+4, 4+3, 8, and 9-10, Lymph node-positive prostate cancer (LN+ Adenocarcinoma), primary small cell neuroendocrine prostate cancer (primary SCNC). The numbers of each samples are 149 of Benign prostate, 73 of Gleason 3+3, 53 of Gleason 3+4, 14 of Gleason 4+3, 3 of Gleason 8, and 8 of Gleason 9-10. (b) A plot of FOXA2 immunohistochemistry staining intensity (from 0 , no staining to 3 , the strongest staining) in the treatment-naive prostate cancers. (c) A plot of Quick-score for FOXA2 (the intensity of staining $\mathrm{x}$ the percentage of staining) in the treatment-naive prostate cancers. Adeno, adenocarcinoma. LN+ Adeno, adenocarcinoma in lymph node. 
cases, staining intensity was scored as 3 in six samples and 1-2 in the remaining two samples (Figure 4b). Our findings indicate that FOXA2 expression could be used to identify the development of treatment-related small cell neuroendocrine carcinoma in patients who relapse after therapy. a

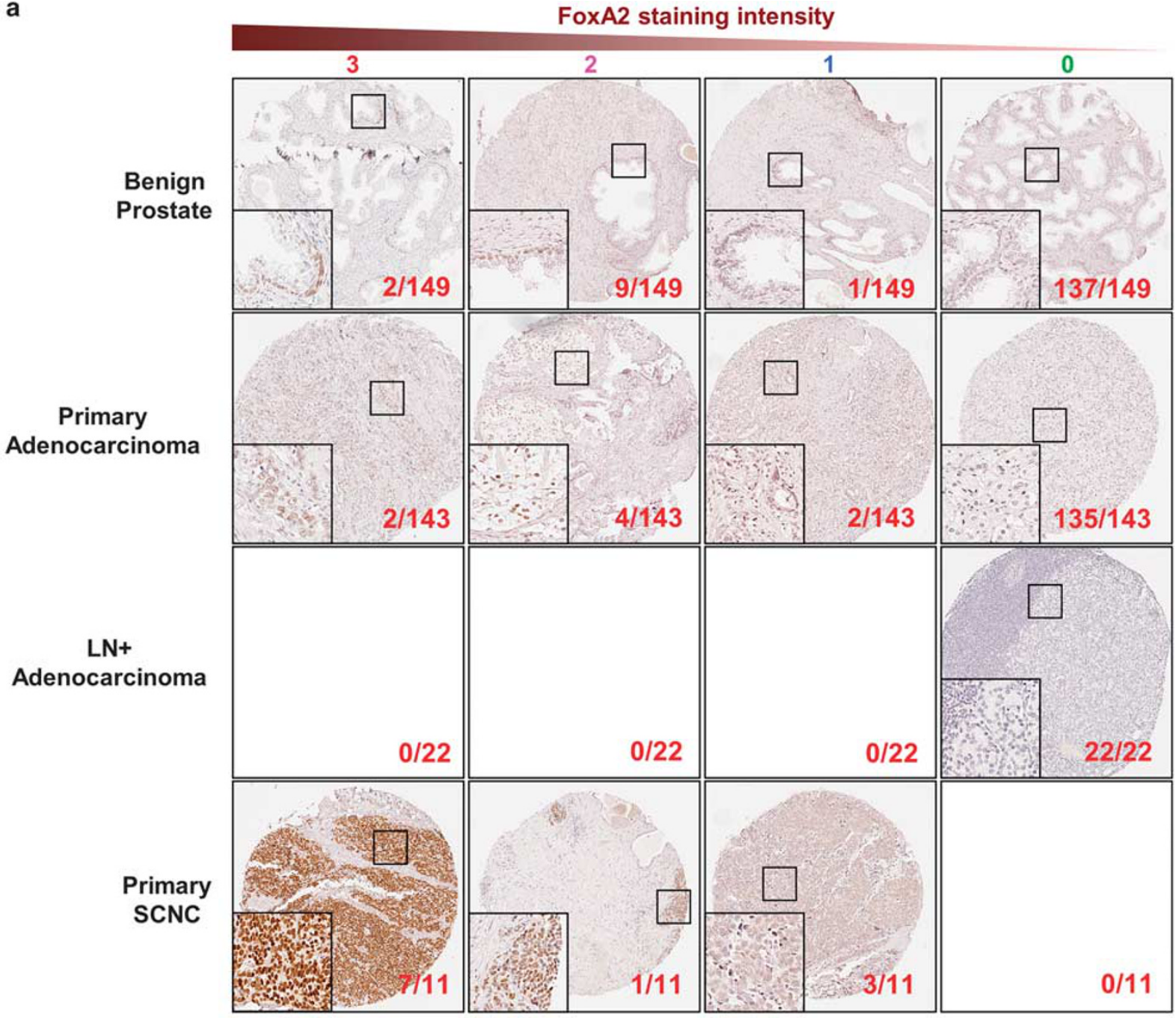

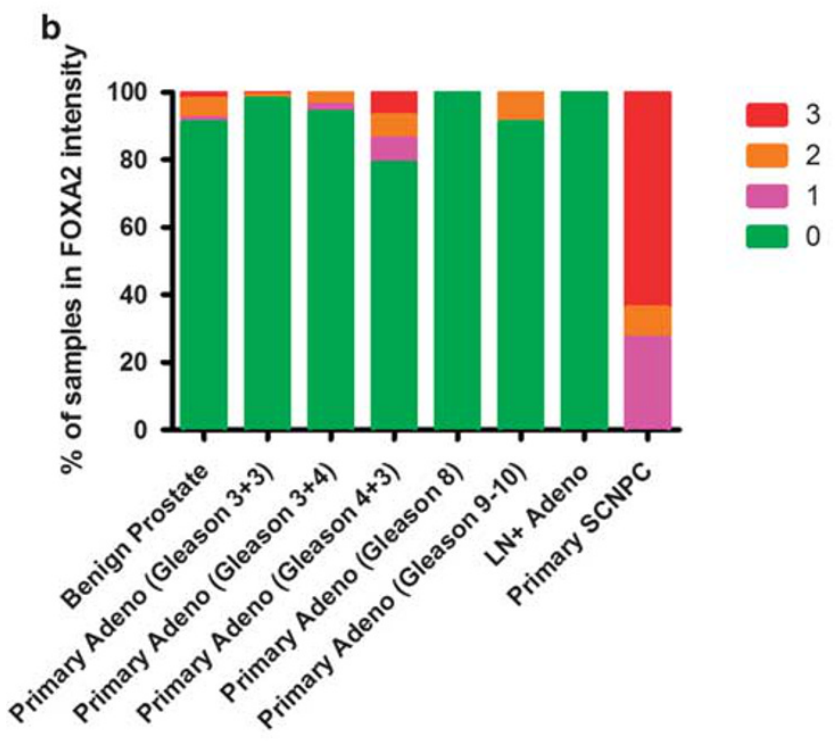

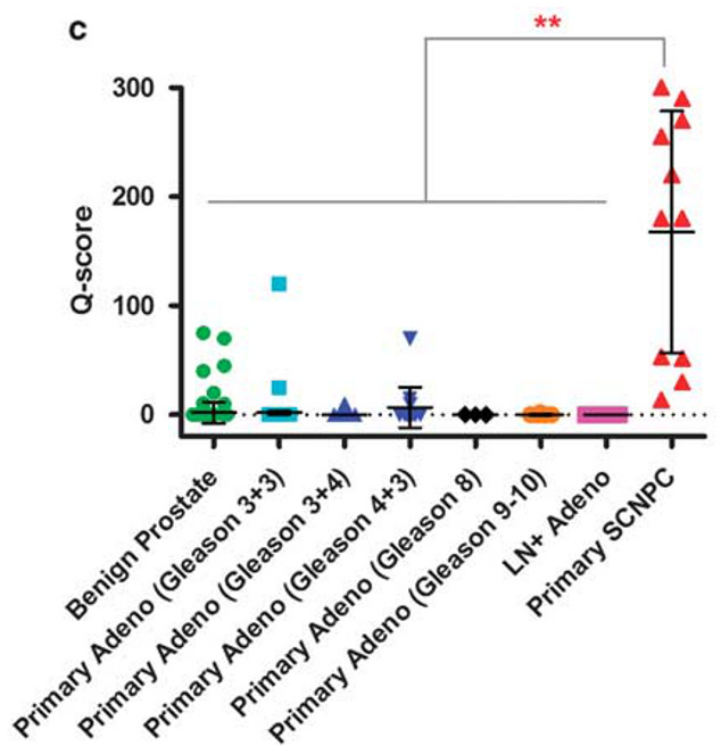

Modern PAthology (2017) 30, 1262-1272 
a

FoxA2 staining intensity

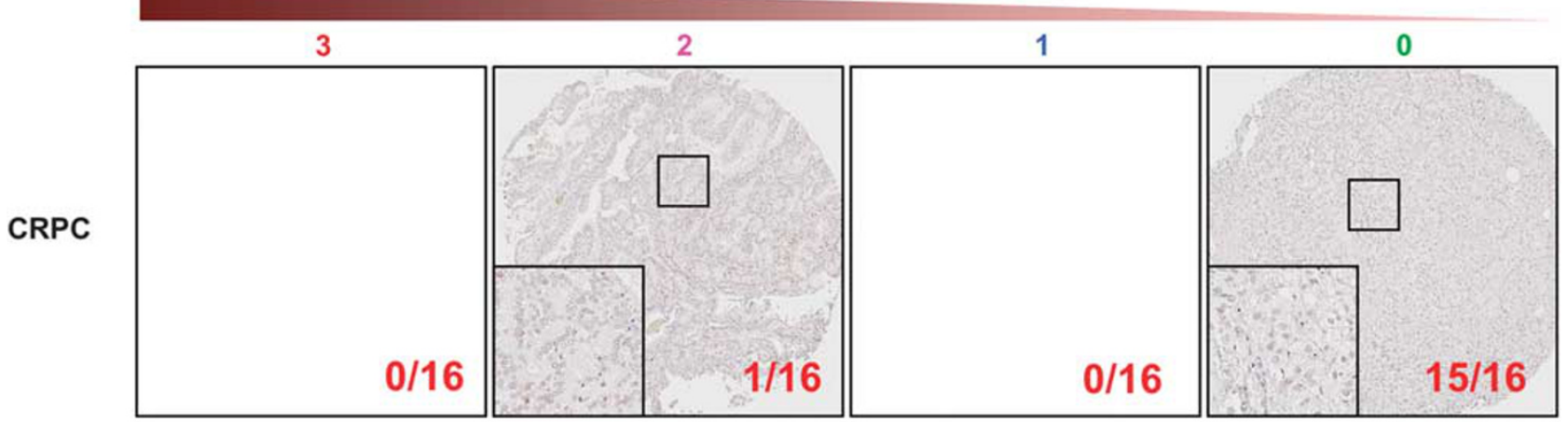

b

Patient \#1
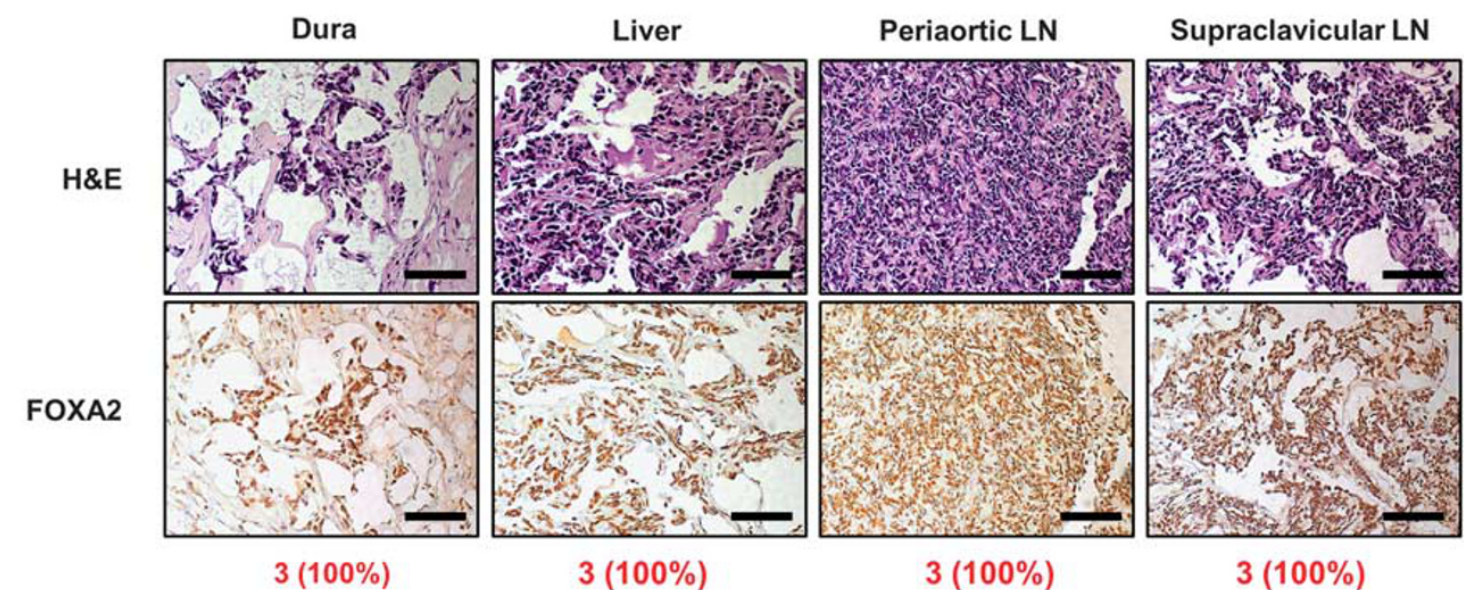

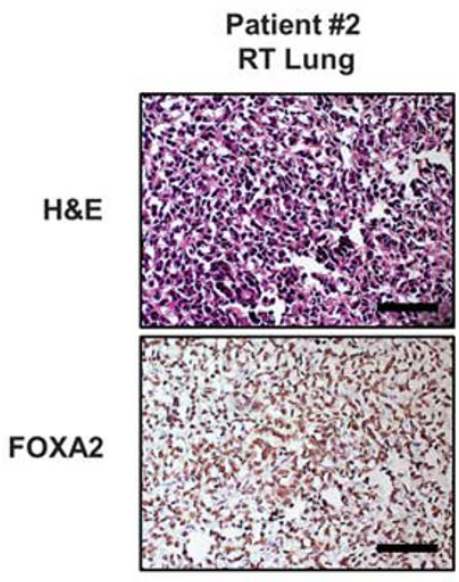

$3(70 \%)$

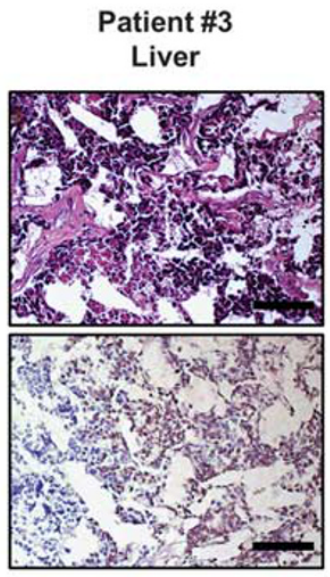

$1(30 \%)$
$3(100 \%)$

Patien \#4

Periaortic LN
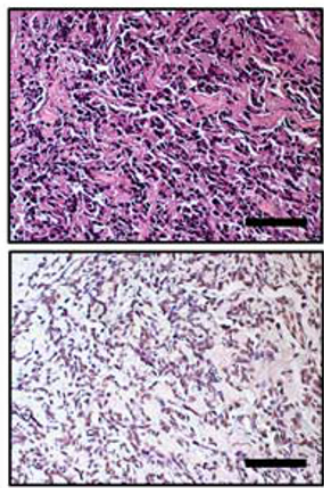

$2(70 \%)$

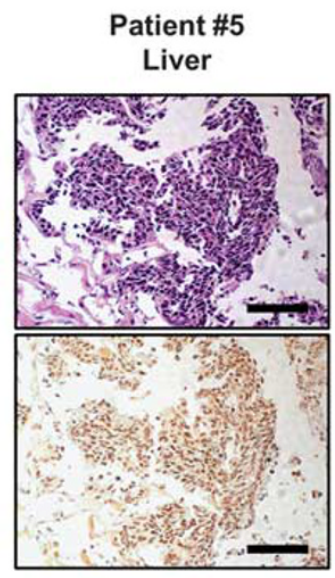

$3(60 \%)$

Figure 4 FOXA2 expression in treatment-resistant prostate cancers. (a) Representative images of FOXA2 immunohistochemistry on organconfined castration-resistant prostate cancer (CRPC, $N=16)$. (b) Hematoxylin and eosin staining and immunohistochemistry of FOXA2 in metastatic castration-resistant prostate cancer specimens from autopsy. The intensity (0, no staining to 3, strongest staining) and the percentage of FOXA2 staining are under the images of FOXA2 staining. LN, lymph node. Scale bar, $100 \mu \mathrm{m}$.

Direct Comparison of FOXA2 with Two Standard Neuroendocrine Markers, Synaptophysin and Chromogranin A in Histologically Identified Small Cell Neuroendocrine Carcinoma

Immunohistochemical evaluation of the expression of two established neuroendocrine markers, chromogranin A and synaptophysin, is commonly used to confirm the clinical diagnosis of small cell neuroendocrine carcinoma. Chromogranin A is a member of the granin family of neuroendocrine secretory proteins and synaptophysin is the major synaptic vesicle protein p38. Given our data suggesting that FOXA2 is a marker of small cell neuroendocrine carcinoma, we next compared the expression of 
FOXA2 with chromogranin A and synaptophysin in a primary small cell neuroendocrine carcinoma tissue microarray (11 samples) and small cell neuroendocrine carcinoma from the rapid autopsy samples (9 samples). Among 20 small cell neuroendocrine carcinoma samples from metastatic castration-resistant prostate cancer and small cell neuroendocrine carcinoma tissue microarray, only five tissues $(25 \%)$ were positive for chromogranin A and eleven tissues (55\%) showed chromogranin A staining (Table 1), whereas 19 small cell neuroendocrine carcinoma samples were positive for FOXA2 (95\%). These results suggest that FOXA2 is superior to existing NE markers and is a highly sensitive and specific biomarker for the detection of small cell neuroendocrine carcinoma.

\section{Evaluation of the Specificity and Sensitivity of Immunohistochemical FOXA2 Staining for the Clinical Diagnosis of Small Cell Neuroendocrine Carcinoma}

Currently, the gold standard for the diagnosis of small cell neuroendocrine carcinoma by surgical pathologists is the identification of unique but subtle histological features that requires highly trained eyes. To rigorously examine the value of FOXA2 expression as a clinical biomarker, we evaluated the sensitivity and specificity of FOXA2 immunohistochemistry staining for the diagnosis of small cell neuroendocrine carcinoma. We evaluated multiple prostate cancer tissues including primary prostate adenocarcinoma, prostate adenocarcinoma with lymph node metastasis, primary small cell neuroendocrine carcinoma, and from metastatic treatmentrelated small cell neuroendocrine carcinoma. Of these, 20 of 235 specimens (8.5\%) showed diagnostic histologic features of small cell neuroendocrine carcinoma as judged histologically. Fifteen of 20 small cell neuroendocrine carcinoma tissues $(75 \%)$ showed strong expression of FOXA2 (staining intensity 2 or 3 ). Four small cell neuroendocrine carcinoma tissues displayed weak expression of FOXA2 (staining intensity 1). We could not detect FOXA2 expression in one of 20 small cell neuroendocrine carcinoma tissues. FOXA2 expression was also detected in 9 of 215 prostate cancer tissues (4.2\%) that were not histologically defined as small cell neuroendocrine carcinoma. FOXA2 staining was able to distinguish the small cell neuroendocrine carcinoma samples among a diverse range of prostate cancer tissues $(P<0.0001$, two-sided Fisher's exact test in Table 2). We considered FOXA2 staining intensity 2 or 3 as positive staining to evaluate the sensitivity and specificity of FOXA2 staining. The sensitivity of FOXA2 staining by immunohistochemistry for small cell neuroendocrine carcinoma was 0.7500 (95\% CI; 0.5090-0.9134) and the specificity was 0.9581 (95\% CI; 0.9220-0.9807) (Table 2). Relative to the reported sensitivity of other established small cell neuroendocrine carcinoma markers, ${ }^{27}$ FOXA2 staining demonstrates a higher sensitivity for small cell neuroendocrine carcinoma and a similar specificity.

\section{Discussion}

The development of highly aggressive small cell neuroendocrine carcinoma in prostate cancer patients undergoing treatment with AR-targeted therapies is a critical clinical issue. The ability to properly monitor

Table 1 Comparison of small cell neuroendocrine carcinoma specific biomarkers

\begin{tabular}{|c|c|c|c|}
\hline Patient ID/tumor location & Chromogranin $A$ & Synaptophysin & FOXA2 \\
\hline Patient \#1/Dura & $\mathrm{N}$ & $\mathrm{P}$ & $\mathrm{P}$ \\
\hline Patient \#1/Liver & $\mathrm{N}$ & $\mathrm{P}$ & $\mathrm{P}$ \\
\hline Patient \#1/Periaortic LN & $\mathrm{N}$ & $\mathrm{N}$ & $\mathrm{P}$ \\
\hline Patient \#1/Supraclavicular LN & $\mathrm{N}$ & $\mathrm{N}$ & $\mathrm{P}$ \\
\hline Patient \#2/RT lung & $\mathrm{N}$ & $\mathrm{N}$ & $\mathrm{P}$ \\
\hline Patient \#3/Liver & $\mathrm{N}$ & $\mathrm{P}$ & $\mathrm{P}$ \\
\hline Patient \#4/Periaortic LN & $\mathrm{N}$ & $\mathrm{P}$ & $\mathrm{P}$ \\
\hline Patient \#5/Liver & $\mathrm{N}$ & $\mathrm{N}$ & $\mathrm{P}$ \\
\hline Patent \#6/Lung & $\mathrm{N}$ & $\mathrm{N}$ & $\mathrm{N}$ \\
\hline SCNC-TMA \#1/Prostate & $\mathrm{N}$ & $\mathrm{N}$ & $\mathrm{P}$ \\
\hline SCNC-TMA \#2/Prostate & $\mathrm{P}$ & $\mathrm{P}$ & $\mathrm{P}$ \\
\hline SCNC-TMA \#3/Prostate & $\mathrm{P}$ & $\mathrm{P}$ & $\mathrm{P}$ \\
\hline SCNC-TMA \#4/Prostate & $\mathrm{N}$ & $\mathrm{N}$ & $\mathrm{P}$ \\
\hline SCNC-TMA \#5/Prostate & $\mathrm{P}$ & $\mathrm{P}$ & $\mathrm{P}$ \\
\hline SCNC-TMA \#6/Prostate & $\mathrm{N}$ & $\mathrm{N}$ & $\mathrm{P}$ \\
\hline SCNC-TMA \#7/Prostate & $\mathrm{P}$ & $\mathrm{P}$ & $\mathrm{P}$ \\
\hline SCNC-TMA \#8/Prostate & $\mathrm{N}$ & $\mathrm{N}$ & $\mathrm{P}$ \\
\hline SCNC-TMA \#9/Prostate & $\mathrm{N}$ & $\mathrm{P}$ & $\mathrm{P}$ \\
\hline SCNC-TMA \#10/Prostate & $\mathrm{N}$ & $\mathrm{P}$ & $\mathrm{P}$ \\
\hline \multirow{2}{*}{ SCNC-TMA \#11/Prostate } & $\mathrm{P}$ & $\mathrm{P}$ & $\mathrm{P}$ \\
\hline & $5 / 20(25 \%)$ & $11 / 20(55 \%)$ & $19 / 20(98 \%)$ \\
\hline
\end{tabular}

Abbreviations: SCNC, small cell neuroendocrine carcinoma; LN, lymph node; TMA, tissue microarray; P, positive staining; N, negative staining. 
Table 2 Comparing a sensitivity and a sensitivity of a new biomarker, FOXA2 for small cell neuroendocrine carcinoma among prostate cancers

\begin{tabular}{lccc} 
& \multicolumn{2}{c}{$\begin{array}{c}\text { Histopathological features of small cell } \\
\text { neuroendocrine carcinoma }\end{array}$} \\
\cline { 2 - 4 } & Positive & Negative & Total \\
\hline FOXA2 expression & & & \\
Positive & 15 & 9 & 24 \\
Negative & 5 & 206 & 211 \\
Total & 20 & 215 & 235
\end{tabular}

Note: Positive FOXA2 expression = staining intensity $2-3+$.

and diagnose small cell neuroendocrine carcinoma is essential in order to stratify patients for appropriate therapy and clinical trial enrollment. Beyond the histological features of small cell neuroendocrine carcinoma which have been well-characterized, the molecular characteristics of small cell neuroendocrine carcinoma remain to be fully elucidated. Recently, next-generation sequencing technologies have identified unique transcriptional profiles in small cell neuroendocrine carcinoma compared with conventional adenocarcinoma. ${ }^{20-22}$ In this study, we evaluated the expression of a differentially expressed pioneer transcription factor, FOXA2, in prostate cancer tissue microarrays of treatment-naive prostate adenocarcinoma, castration-resistant prostate adenocarcinoma, primary small cell neuroendocrine carcinoma and treatment-related metastatic small cell neuroendocrine carcinoma. Remarkably, FOXA2 expression was detected specifically in small cell neuroendocrine carcinoma, both in primary cases and in those that evolved after hormonal therapy of prostate adenocarcinoma. Currently, the two neuroendocrine differentiation markers, synaptophysin and chromogranin A are used to confirm the diagnosis of small cell neuroendocrine carcinoma called by pathologists. However, these markers are frequently detected in prostate adenocarcinoma (10$40 \%) .{ }^{28}$ Importantly, our findings show that the specificity of FOXA2 staining (95.8\% in Table 2 ) in our large cohorts is higher than the previously reported specificity of synaptophysin or chromogranin $A .{ }^{28,29}$ In addition, we directly compared the performance of FOXA2 positivity by immunohistochemistry to those of synaptophysin and chromogranin A in our tissue samples and show that FOXA2 is a far more sensitive marker for the diagnosis of small cell neuroendocrine carcinoma. Evaluation of FOXA2 expression by immunohistochemical analysis is, therefore, a valuable tool to assist pathologists in the accurate diagnosis of small cell neuroendocrine carcinoma. Our findings strongly suggest that FOXA2 expression can distinguish small cell neuroendocrine carcinoma from treatment-naive and -resistant prostate adenocarcinoma regardless of metastatic status. However, other previously reported small cell neuroendocrine carcinoma biomarkers such as TTF-1 and Ki67, ${ }^{27,29}$ may provide additional power when combined with FOXA2 to confirm a diagnosis of small cell neuroendocrine carcinoma during difficult cases. Also, a recent study suggested that loss of cyclin D1 expression may help to identify small cell neuroendocrine carcinoma and a small subset of adenocarcinomas with poor prognosis. ${ }^{30}$

During mouse prostate development, FOXA2 is expressed in embryonic prostate buds but disappears in the adult prostate. ${ }^{10}$ We have shown that expression of FOXA2 is a sensitive and specific marker of small cell neuroendocrine carcinoma. However, the function of FOXA2 in the pathogenesis of small cell neuroendocrine carcinoma is unclear. A recent study provided evidence that FOXA2 is co-localized with and directly interacts with HIF-1a in the TRAMP model. ${ }^{31}$ The HIF1 $\alpha$-FOXA2 complex induced the transcription of genes that are important for tumorigenesis in a cell line derived from the TRAMP model. ${ }^{31}$ Another study using the TRAMP model showed that the expression of the pro-neural transcription factor ASCL1 was increased by knocking out FOXA2, suggesting negative regulation of ASCL1 by FOXA2. ${ }^{32}$ In addition, it has been reported that FOXA2 can be induced by active Wnt/ $\beta$-catenin signaling, and the expression of FOXA2 was associated with an invasive phenotype in primary prostate cancer. ${ }^{33,34}$

Among the various histologic types of lung cancer, FOXA2 expression is also detected in most neuroendocrine tumors including typical carcinoids, atypical carcinoids, large cell neuroendocrine carcinomas, and small cell carcinomas, while non-neuroendocrine tumors such as adenocarcinomas and squamous cell carcinomas lack or show low levels of FOXA2 expression. ${ }^{35}$ The finding of FOXA2 expression in neuroendocrine lung cancers and prostate small cell neuroendocrine carcinoma suggests that FOXA2 may be a common marker of neuroendocrine cancer differentiation in epithelial tissues.

In summary, we have shown that FOXA2 is a more specific and sensitive molecular marker for small cell neuroendocrine carcinoma than currently used neuroendocrine markers, synaptophysin and chromogranin A. Thus, FOXA2 immunohistochemistry will be useful to confirm a diagnosis of small cell neuroendocrine carcinoma even for pathologists who do not have experience with many cases of small cell neuroendocrine carcinoma. Plus, FOXA2 staining will be useful to monitor cancer progression from adenocarcinoma to small cell neuroendocrine carcinoma in patients. Our findings need to be validated in different patient cohorts by independent laboratory groups to verify the clinical utility of FOXA2 staining. Future studies will be directed at understanding the biologic function of FOXA2 and unveiling the role of FOXA2 in the evolution of small cell neuroendocrine carcinoma. 


\section{Acknowledgments}

J.W. Park is supported by the Eli and Edythe Broad Center of Regenerative Medicine and Stem Cell Research at the University of California, Los Angeles (UCLA) Training Program; J.K.L. is supported by the Tower Cancer Research Foundation Career Development Award, a Prostate Cancer Foundation Young Investigator Award, and the UCLA Subspecialty Training and Advanced Research Program; J.H. is supported by the National Institutes of Health (Grants 1R01CA181242, 5R01CA172603, and 1R01CA205001), the Department of Defense Prostate Cancer Research Program (PC150382 and PC150382); O.N.W. is an Investigator of the Howard Hughes Medical Institute supported by National Institutes of Health Grant U01 CA164188-01A and a Prostate Cancer Foundation Challenge Award; J.H. and O.N.W. are supported by Stand Up To Cancer/ Prostate Cancer Foundation/Prostate Dream Team Translational Cancer Research Grant SU2C-AACRDT0812. This research grant is made possible by the generous support of the Movember Foundation. Stand Up To Cancer is a program of the Entertainment Industry Foundation administered by the American Association for Cancer Research.

\section{Disclosure/conflict of interest}

The authors declare no conflict of interest.

\section{References}

1 Helpap B, Kollermann J, Oehler U. Neuroendocrine differentiation in prostatic carcinomas: histogenesis, biology, clinical relevance, and future therapeutical perspectives. Urol Int 1999;62:133-138.

2 Miyoshi Y, Uemura H, Kitami K, et al. Neuroendocrine differentiated small cell carcinoma presenting as recurrent prostate cancer after androgen deprivation therapy. BJU Int 2001;88:982-983.

3 Tanaka M, Suzuki Y, Takaoka K, et al. Progression of prostate cancer to neuroendocrine cell tumor. Int J Urol 2001;8:431-436.

4 Turbat-Herrera EA, Herrera GA, Gore I, et al. Neuroendocrine differentiation in prostatic carcinomas. A retrospective autopsy study. Arch Pathol Lab Med 1988;112:1100-1105.

5 Wang HT, Yao YH, Li BG, et al. Neuroendocrine Prostate Cancer (NEPC) progressing from conventional prostatic adenocarcinoma: factors associated with time to development of NEPC and survival from NEPC diagnosis-a systematic review and pooled analysis. J Clin Oncol 2014;32:3383-3390.

6 Nadal R, Schweizer M, Kryvenko ON, et al. Small cell carcinoma of the prostate. Nat Rev Urol 2014;11:213-219.

7 Sun Y, Niu J, Huang J. Neuroendocrine differentiation in prostate cancer. Am J Transl Res 2009;1:148-162.

8 Chiaverotti T, Couto SS, Donjacour A, et al. Dissociation of epithelial and neuroendocrine carcinoma lineages in the transgenic adenocarcinoma of mouse prostate model of prostate cancer. Am J Pathol 2008;172:236-246.

9 Lee JK, Phillips JW, Smith BA, et al. N-Myc drives neuroendocrine prostate cancer initiated from human prostate epithelial cells. Cancer Cell 2016;29:536-547.

10 Mirosevich J, Gao N, Matusik RJ. Expression of Foxa transcription factors in the developing and adult murine prostate. Prostate 2005;62:339-352.

11 Mirosevich J, Gao N, Gupta A, et al. Expression and role of Foxa proteins in prostate cancer. Prostate 2006;66:1013-1028.

12 Hansson J, Abrahamsson PA. Neuroendocrine pathogenesis in adenocarcinoma of the prostate. Ann Oncol 2001;12:S145-S152.

13 Evangelou AI, Winter SF, Huss WJ, et al. Steroid hormones, polypeptide growth factors, hormone refractory prostate cancer, and the neuroendocrine phenotype. J Cell Biochem 2004;91:671-683.

14 Huang J, Yao JL, Zhang L, et al. Differential expression of interleukin-8 and its receptors in the neuroendocrine and non-neuroendocrine compartments of prostate cancer. Am J Pathol 2005;166:1807-1815.

15 Zhang Z, Rosen DG, Yao JL, et al. Expression of p14ARF, p15INK4b, p16INK4a, and DCR2 increases during prostate cancer progression. Mod Pathol 2006;19:1339-1343.

16 Chen R, Zeng X, Zhang R, et al. Cav1.3 channel alpha1D protein is overexpressed and modulates androgen receptor transactivation in prostate cancers. Urol Oncol 2014;32:524-536.

17 Qin J, Liu X, Laffin B, et al. The PSA(-/lo) prostate cancer cell population harbors self-renewing long-term tumor-propagating cells that resist castration. Cell Stem Cell 2012;10:556-569.

18 Harvey JM, Clark GM, Osborne CK, et al. Estrogen receptor status by immunohistochemistry is superior to the ligand-binding assay for predicting response to adjuvant endocrine therapy in breast cancer. J Clin Oncol 1999;17:1474-1481.

19 Shultz LD, Ishikawa F, Greiner DL. Humanized mice in translational biomedical research. Nat Rev Immunol 2007;7:118-130.

20 Beltran H, Rickman DS, Park K, et al. Molecular characterization of neuroendocrine prostate cancer and identification of new drug targets. Cancer Discov 2011;1:487-495.

21 Zhang X, Coleman IM, Brown LG, et al. SRRM4 expression and the loss of REST activity may promote the emergence of the neuroendocrine phenotype in castration-resistant prostate cancer. Clin Cancer Res 2015;21:4698-4708.

22 Beltran H, Prandi D, Mosquera JM, et al. Divergent clonal evolution of castration-resistant neuroendocrine prostate cancer. Nat Med 2016;22:298-305.

23 Akamatsu S, Wyatt AW, Lin D, et al. The Placental Gene PEG10 promotes progression of neuroendocrine prostate cancer. Cell Rep 2015;12:922-936.

24 Tai S, Sun Y, Squires JM, et al. PC3 is a cell line characteristic of prostatic small cell carcinoma. Prostate 2011;71:1668-1679.

25 Mertz KD, Setlur SR, Dhanasekaran SM, et al. Molecular characterization of TMPRSS2-ERG gene fusion in the NCI-H660 prostate cancer cell line: a new perspective for an old model. Neoplasia 2007;9: 200-206.

26 Bello D, Webber MM, Kleinman HK, et al. Androgen responsive adult human prostatic epithelial cell lines 
immortalized by human papillomavirus 18. Carcinogenesis 1997;18:1215-1223.

27 Yao JL, Madeb R, Bourne P, et al. Small cell carcinoma of the prostate: an immunohistochemical study. Am J Surg Pathol 2006;30:705-712.

28 Ather $\mathrm{MH}$, Abbas F, Faruqui N, et al. Correlation of three immunohistochemically detected markers of neuroendocrine differentiation with clinical predictors of disease progression in prostate cancer. BMC Urol 2008;8:21.

29 Wang W, Epstein JI. Small cell carcinoma of the prostate. A morphologic and immunohistochemical study of 95 cases. Am J Surg Pathol 2008;32:65-71.

30 Tsai H, Morais CL, Alshalalfa M, et al. Cyclin D1 loss distinguishes prostatic small-cell carcinoma from most prostatic adenocarcinomas. Clin Cancer Res 2015;21: 5619-5629.
31 Qi J, Nakayama K, Cardiff RD, et al. Siah2-dependent concerted activity of HIF and FoxA2 regulates formation of neuroendocrine phenotype and neuroendocrine prostate tumors. Cancer Cell 2010;18:23-38.

32 Gupta A, Yu X, Case T, et al. Mash1 expression is induced in neuroendocrine prostate cancer upon the loss of Foxa2. Prostate 2013;73:582-589.

$33 \mathrm{Yu}$ X, Wang Y, DeGraff DJ, et al. Wnt/beta-catenin activation promotes prostate tumor progression in a mouse model. Oncogene 2011;30:1868-1879.

$34 \mathrm{Yu} \mathrm{X}$, Wang Y, Jiang M, et al. Activation of beta-Catenin in mouse prostate causes HGPIN and continuous prostate growth after castration. Prostate 2009;69:249-262.

35 Khoor A, Stahlman MT, Johnson JM, et al. Forkhead box A2 transcription factor is expressed in all types of neuroendocrine lung tumors. Hum Pathol 2004;35: $560-564$.

Supplementary Information accompanies the paper on Modern Pathology website (http://www.nature.com/ modpathol) 